\title{
Aumento da imunorreatividade ao VEGFR-1 no complexo coroido-escleral em modelo experimental de hipercolesterolemia
}

\author{
Increased VEGFR-1 immunoreactivity in the choroid-scleral complex in hypercholesterolemia \\ experimental model
}

Rogil José de Almeida Torres ${ }^{1}$, Lucia de Noronha², Antonio Marcelo Barbante Casella ${ }^{3}$, Regiane do Rocio de Almeida Torres ${ }^{4}$, Isabela de Carvalho Martins ${ }^{5}$, Rafael Zotz ${ }^{5}$, Andréa Luchinl ${ }^{6}$, Conrado Roberto Hoffmann Filho ${ }^{7}$, Dalton Bertolim Précoma ${ }^{8}$

\section{RESUMO}

Objetivo: $O$ objetivo deste trabalho é investigar a expressão do fator de crescimento vascular endotelial (VEGF) na coroide e esclera, utilizando um modelo experimental de hipercolesterolemia.

Método: Coelhos New Zealand foram organizados em dois grupos: $\mathrm{O}$ grupo dieta normal (GN), composto por 8 coelhos (8 olhos), recebeu ração padrão para coelhos, durante 4 semanas; e o grupo hipercolesterolêmico $(\mathrm{GH})$, composto por 13 coelhos (13 olhos), recebeu dieta rica em colesterol a 1\% por 8 semanas. Foi realizada a dosagem sérica de colesterol total, triglicerídeos, HDL colesterol, glicemia de jejum no início do experimento e no momento da eutanásia. Ao final da 8 a semana para - GH e 4a semana para o GN foi realizada a eutanásia dos animais e os olhos foram submetidos à análise imuno-histoquímica com os anticorpos RAM-11 eVEGFR-1. Resultados: Observou-se significativo aumento do colesterol total e triglicerídeos do $\mathrm{GH}$ em relação ao $G N(p<0,001)$. Houve significativo aumento da expressão da RAM-11 e VEGFR-1 na coroide e esclera dos animais do GH em relação ao GN $(p \leq 0,001)$.

Conclusão: Este estudo demonstra que a dieta hipercolesterolêmica em coelhos induz ao aumento da concentração de macrófagos e da imunorreatividade ao VEGFR-1 na coroide e esclera, expressando similaridade com a degeneração macular relacionada à idade (DMRI) humana.

Descritores: Fator A de crescimento do endotélio vascular; Degeneração macular: Macrófagos; Colesterol; Coroide; Esclera; Animais; Coelhos

\section{ABSTRACT}

Purpose: The aim of this study is to investigate the expression of vascular endothelial growth factor (VEGF) in the choroid and sclera using hypercholesterolemia experimental model.

Methods: New Zealand rabbits were divided into two groups: 8 rabbits ( 8 eyes), in the normal diet group (NG), were fed by a standard diet for 4 weeks; and 13 rabbits (13 eyes), in the hypercholesterolemic group (HG), were fed by a $1 \%$ cholesterol-enriched diet for 8 weeks. Total serum cholesterol, triglyceride, HDL cholesterol and fasting blood glucose exams were performed at the initiation of the experiment and at the euthanasia time. After hypercholesterolemic group $8^{\text {th }}$ week and NG $4^{\text {th }}$ week, animals were euthanized and their eyes underwent immunohistochemical analysis with the RAM-11 and VEGFR-1).

Results: The diet has induced a significant increase in total cholesterol and triglyceride levels in HG when compared with NG ( $p<0.001)$. There was a significant increase in the RAM-11 and VEGFR-1 expressions in hypercholesterolemic group choroid and sclera in relation to $N G(p \leq 0,001)$.

Conclusion: This study has revealed that the hypercholesterolemic diet in rabbits induces an increase in the macrophage concentration and immunoreactivity to VEGFR-1 in the choroid and sclera, resembling human age-related maculardegeneration (ARMD).

Keywords: Vascularendothelial growth factor A; Macular degeneration; Macrophages; Cholesterol; Choroid; Sclera; Animals; Rabbits

\section{INTRODUÇÃO}

Degeneração macular relacionada à idade (DMRI) é uma das principais causas de perda irreversível da visão na terceira idade ${ }^{(1)}$. É uma doença complexa e multifatorial associada ao envelhecimento, alterações genéticas e ambientais ${ }^{(2)}$. Estudos histopatológicos demonstram que o epitélio pigmentário da retina (EPR), membrana de Bruch (MB) e coriocapilar estão envolvidos primariamente neste processo patológico(3). Sugere-se que o envelhecimento induza a gradativa deposição anômala de lipídeos na $\mathrm{MB}^{(3)}$, levando ao aumento de sua espessura ${ }^{(4)}$. A MB é um tecido conectivo fino e semipermeável que permite a passagem de nutrientes da coriocapilar para o EPR e camadas externas da retina sensorial. Esta estrutura também dá passagem aos metabólitos produzidos pelas células do EPR para a coriocapilar. Sendo assim, a modificação da MB interfere na nutrição das células do EPR conduzindo-as a gradativo processo de atrofia(3,5,6). Uma vez estabelecido o processo atrófico do EPR, há redução da necessidade de suprimento sanguíneo para esta camada, provocando atrofia secundária da coriocapilar ${ }^{(4,7,8)}$. Desta forma, as camadas externas da
Submetido para publicação: 9 de fevereiro de 2012

Aceito para publicação: 13 de novembro de 2012

Trabalho realizado na Pontifícia Universidade Católica do Paraná.

Médico, Departamento de Oftalmologia, Pontifícia Universidade Católica do Paraná - PUCPR Curitiba (PR), Brasil.

Médica, Departamento de Patologia, Pontifícia Universidade Católica do Paraná - PUCPR - Curitiba (PR), Brasil.

${ }^{3}$ Médico, Departamento de Oftalmologia, Universidade Estadual de Londrina - UEL - Londrina (PR), Brasil.

${ }^{4}$ Acadêmica de Medicina, Pontifícia Universidade Católica do Paraná - PUCPR - Curitiba (PR), Brasil.

Zootecnista, Biotério Central, Pontifícia Universidade Católica do Paraná - PUCPR - Curitiba (PR), Brasil.

Médica, Centro Oftalmológico de Curitiba, Curitiba (PR), Brasil.

Médico, Departamento de Cirurgia, Pontifícia Universidade Católica do Paraná - PUCPR - Curitiba

Paraná, Brasil.

Professor Titular, Departamento de Cardiologia, Pontifícia Universidade Católica do Paraná -

PUCPR - Curitiba (PR), Brasil.
Financiamento: Não houve financiamento para este trabalho.

Divulgação de potenciais conflitos de interesse: R.J.A.Torres, Nenhum; L.de Noronha, Nenhum; A.M.B.Casella, Nenhum; R.R.A.Torres, Nenhum; I.C.Martins, Nenhum; R.Zotz, Nenhum; A.Luchini, Nenhum; C.R.Hoffmann Filho, Nenhum; D.B.Précoma, Nenhum.

Endereço de correspondência: Rogil José de Almeida Torres. Rua Emiliano Perneta 390 - Conj. 1407 Curitiba (PR) - 80420-080 - Brasil - E-mail: rjat@terra.com.br

Projeto número 240/080. CEUA - Pontifícia Universidade Católica do Paraná.

Registro do parecer no CEUA: 307

Data do parecer: 19/08/2008 
retina sensorial, o EPR e a coriocapilar entram em hipóxia ${ }^{(9,10)}$. Sabe-se que a hipóxia é o principal estímulo para liberação do fator de crescimento endotelial vascular (VEGF)(11), responsável pela formação de neovasos sub-retinianos ${ }^{(2)}$

Clinicamente, a perda da visão central na DMRI pode ocorrer pela atrofia geográfica de coroide, denominada DMRI seca, e/ou pelo desenvolvimento de membrana neovascular sub-retiniana, denominada DMRI úmida. A DMRI seca reduz progressivamente e lentamente a acuidade visual (AV). Até o momento, preconiza-se o uso de antioxidantes sintéticos, dieta antioxidante e mudança de hábitos de vida para estabilizar ou atenuar o avanço da atrofia geográfica da coroide ${ }^{(12)}$. Por outro lado, a DMRI úmida pode provocar metamorfopsia e queda abrupta da visão. Atualmente, o principal alvo terapêutico da DMRI úmida é o VEGF, maior estimulador de neovascularização ocular ${ }^{(2,13)}$. Várias drogas têm sido utilizadas para neutralizar este fator de crescimento angiogênico com o intuito de promover a regressão da neovascularização sub-retiniana e manter a visão central, porém o prognóstico visual permanece ruim ${ }^{(14)}$.

Sendo assim, o conhecimento de fatores que estimulam a produção do VEGF é importante para a prevenção da doença macular degenerativa. Desta forma, a criação de modelos experimentais que possam induzir a liberação deste fator de crescimento vascular aprimora o conhecimento fisiopatogênico desta temível doença e propicia pesquisas terapêuticas experimentais que podem ser úteis futuramente no combate à DMRI.

O objetivo deste trabalho é investigar a expressão do VEGF na coroide e esclera, utilizando um modelo experimental de hipercolesterolemia.

\section{MÉTODOS}

Para a realização deste estudo, o protocolo foi aprovado pela Comissão de Ética em Experimentação Animal da Pontifícia Universidade Católica do Paraná, seguindo os princípios da Declaração de Helsinque (1964).

\section{Ambiente de EXPERIMENTAÇÃo}

Os procedimentos deste estudo foram realizados nas dependências do laboratório de Técnica Operatória da PUC-PR e do Centro de Estudos do Hospital Angelina Caron (HAC). Os animais foram mantidos no biotério em macroambiente com ciclos de iluminação $12 / 12$ horas, com trocas de ar e temperatura controlada de 19 a $23^{\circ} \mathrm{C}$ e receberam durante o experimento água e ração específica para a espécie Nuvital ${ }^{\circledR}$ (Nuvital, Colombo, Brasil) de forma ad libitum.

\section{Animais Utilizados E Delineamento eXPERIMENTAL}

Foram utilizados 21 coelhos machos albinos (Oryctolagus cunicullus), da linhagem New Zealand, procedentes do Biotério Central da Pontifícia Universidade Católica do Paraná, com idade média aproximada de 110 dias e peso médio de 2.770 gramas. Os animais foram divididos em dois grupos: grupo 1 (GN), composto por 8 coelhos, e grupo 2 (GH) composto por 13 coelhos. O GN, ou grupo de deita normal, recebeu ração padrão para coelhos de laboratório Nuvital ${ }^{\circledR}(\mathrm{Nu}$ vital, Colombo, Brasil) e foi submetido à eutanásia em quatro semanas. O GH, ou grupo hipercolesterolêmico, recebeu ração padrão para coelhos de laboratório Nuvital ${ }^{\circledR}$ (Nuvital, Colombo, Brasil), acrescida de colesterol a 1\%, em todo o período do estudo (ração suplementar). Este grupo foi submetido à eutanásia ao final de oito semanas.

Cada coelho foi submetido à dosagem sérica de colesterol total, triglicerídeos, HDL colesterol, glicemia de jejum no início do experimento e no momento da eutanásia. As coletas das amostras sanguíneas foram realizadas através de punção da veia marginal auricular magna sob anestesia geral com injeção intramuscular de ketamina $5 \mathrm{mg} / \mathrm{kg}$ e xylazina $35 \mathrm{mg} / \mathrm{kg}$. As dosagens plasmáticas de glicemia, colesterol total, colesterol HDL e triglicerídeos foram realizados pelo método enzimático colorimétrico automatizado (Architec ${ }^{\circledR}$ - Abbott). Uma soroteca com 500 microlitros de cada animal foi congelada para análises futuras. O peso dos coelhos foi aferido em rotina semanal.

Os animais foram sacrificados com injeção endovenosa de $5 \mathrm{ml}$ de pentobarbital e os olhos imediatamente fixados em paraformaldeído a 4\% (Merck, Darmstadt, Germany), em 0,1 M fosfato/pH 7,4 por 4 horas, para análise imuno-histoquímica.

\section{Preparo da ração suplementar}

A ração hipercolesterolêmica a 1\%, utilizada nas oito semanas do experimento, apresentava 200 gramas de colesterol (Sigma-Aldrich à $95 \%{ }^{\circledR}$ ) dissolvido em 800 mililitros de clorofórmio (Biotec ${ }^{\circledR}$ ), distribuídos homogeneamente em 20 quilogramas de ração Nuvital ${ }^{\circledR}$ (Nuvital, Colombo, Brasil). Antes de administrar a ração aos coelhos, esperou-se um período mínimo de 24 horas para adequada evaporação do clorofórmio. A quantidade diária ofertada para cada animal foi de 600 gramas ao dia(15).

\section{Preparação do tecido e anÁlise imuno-histoquímica}

Os dois olhos de cada animal (total de 42) foram removidos e submetidos à fixação, porém somente um foi escolhido para o estudo, de forma aleatória. Depois da fixação os espécimes foram avaliados macroscopicamente, sendo feito uma secção axial ao nível do nervo óptico, dividindo os bulbos oculares em duas metades iguais (superior e inferior). A metade inferior foi estocada para estudos posteriores. Já a metade superior foi submetida à desidratação, diafanização e impregnação em parafina, com histotécnico da marca Leica ${ }^{\circledR}$ (Leica, Wetzlar, Germany), modelo TP 1020. Para a confecção dos blocos de parafina utilizou-se o inclusor Leica ${ }^{\circledR}$, modelo EG1 160. Estes blocos foram cortados com micrótomo, marca Leica ${ }^{\circledR}$ modelo RM2145 (Leica, Wetzlar, Germany), a 5 u para obtenção dos cortes histológicos (total de 42). Estes cortes foram pescados em lâmina de vidro com albumina, corados com hematoxilina-eosina e montados com lamínula de vidro de 24x90 mm Entellan, Merck ${ }^{\circledR}$ (Merck, Darmstadt, Germany).

Os cortes histológicos foram desparafinizados e re-hidratados, sendo feito posteriormente o bloqueio da peroxidase endógena. Posteriormente foram lavados em água deionizada sendo incubados em câmara úmida a $95^{\circ} \mathrm{C}$ por 20 minutos para recuperação antigênica. Após esta fase, foi realizado novo bloqueio da peroxidase endógena. Os cortes foram cobertos pelo anticorpo primário monoclonal produzido em camundongo VEGF receptor 1 (VEGFR 1), da marca THERMOscientific ${ }^{\circledR}$ (diluição 1:100) e pelo anticorpo monoclonal primário RAM-11, da marca Dako ${ }^{\circledR}$ (DakoCytomation, Carpinteria, CA, EUA), na diluição 1/400. Posteriormente, foi recoberto com anticorpo secundário, polímero marcado-HRP anti-camundongo advance ${ }^{\circledR}$ System (DakoCytomation, Inc., Carpinteria, CA) e incubados, à temperatura ambiente, por 30 minutos. A seguir foram submetidos ao gotejamento de substrato misto recém preparado DAB (DakoCytomation, Inc., CA, USA). Novamente foram incubados por 3 até 5 minutos. Os cortes foram contra-corados com hematoxilina de Mayer e depois montados.

Controles positivos e negativos foram usados em todas as marcações e as lâminas foram primeiramente analisadas por um observador, sem conhecimento prévio do grupo de identificação (análise mascarada). Nesta análise, foi anotada a presença ou não de positividade para os marcadores VEGFR 1 e RAM 11. As áreas positivas adquiriram coloração acastanhada e foram analisadas pelo método da morfometria de cores. Para tanto, foram capturadas imagens de cinco campos consecutivos, de pars plana a pars plana contralateral, em objetiva de 40x, utilizando a câmera Olympus BX50, modelo DXC-107A e o software Image Pró Plus. Tal software permitiu que as áreas positivas fossem selecionadas e coloridas por um observador. A área imunorreativa foi automaticamente calculada pelo software e expressa em micrômetros ${ }^{2}$. Estes dados foram registrados no programa Microsoft Excel (Redmond, WA) em forma de planilhas para 
análise estatística. A variável área imunorreativa refere-se à somatória de todas as áreas positivas em cada um dos cinco campos analisados.

\section{Análise estatística}

Para a comparação dos grupos definidos pelo tratamento em relação às variáveis quantitativas, foi considerado o teste t de Student para amostras independentes. Para a comparação entre as avaliações início e eutanásia dentro de cada grupo, foi usado o teste t de Student para amostras pareadas. A condição de normalidade foi avaliada pelo teste de Shapiro-Wilks. Variáveis que não apresentaram a condição de simetria foram submetidas a uma transformação logarítmica. Valores de $p<0,05$ indicaram significância estatística. Os dados foram analisados com o programa computacional Statistica v.8.0.

\section{RESULTADOS}

\section{CoMPARAÇÃo doS GRUPOS EM RELAÇÃo Às VARIÁVEIS: GLICOSE, COLESTEROL TOTAL, HDL E TRIGLICERÍDEOS}

O grupo dieta normal (GN) manteve as variáveis laboratoriais estáveis e normais do início ao fim do experimento. Por outro lado, os animais do GH (grupo dieta hipercolesterolêmica) manifestaram expressivo aumento do colesterol total na eutanásia. No momento basal, a média do colesterol total dos grupos era de aproximadamente $41,3 \mathrm{mg} / \mathrm{dL}$ e ao final observou-se significativa elevação para $2146,8 \mathrm{mg} / \mathrm{dL}(\mathrm{p}<0,001)$, no GH. Outra variável laboratorial que demonstrou importante variação no GH foram os triglicerídeos. No momento basal, a média dos triglicerídeos dos grupos foi de aproximadamente $46,5 \mathrm{mg} / \mathrm{dL}$, enquanto que na eutanásia chegou a $168,5 \mathrm{mg} / \mathrm{dL}(\mathrm{p}=0,001)$, no GH. A glicemia e o HDL colesterol não manifestaram variações significativas ao final do experimento neste grupo.

\section{COMPARAÇÃO DOS GRUPOS EM RELAÇÃO À ÁREA TOTAL IMUNORREATIVA AO RAM-11}

Houve aumento significativo da expressão do RAM-11 na coroide e esclera dos animais do GH (Tabela 1), indicando grande concentração de macrófagos nestas estruturas. A figura 1 demonstra 0 significativo aumento da espessura da coroide e esclera do GH (A) em relação ao GN (B). É possível verificar também que a imunorreatividade coroidal e escleral ao RAM-11 do GH foi superior ao GN. As áreas imunorreativas adquiriram coloração acastanhada.

\section{COMPARAÇÃO dOS GRUPOS EM RELAÇÃo À ÁREA TOTAL IMUNORREATIVA AO VEGFR-1}

Houve aumento significativo da expressão do "vascular endothelial growth factor receptor" (VEGFR-1) na coroide e esclera dos animais do GH (Tabela 2). A figura 2 demonstra o significativo aumento da espessura da coroide e esclera do GH (A) em relação ao GN (B). É possível verificar também que a imunorreatividade coroidal e escleral ao VEGFR-1 do GH foi superior ao GN. As áreas imunorreativas adquiriram coloração acastanhada.

\section{DISCUSSÃO}

Sabe-se que a hipóxia provoca crescimento de vasos pela sinalização de fatores de transcrição, sendo a regulação do VEGF a mais notável, aumentando até 30 vezes em minutos ${ }^{(16)}$. O VEGF estimula células endoteliais a degradar a matriz extracelular, proliferar, migrar e formar condutos, agindo como fator de sobrevivência ${ }^{(17)}$. A ação principal do VEGF se dá nos capilares venosos, conduzindo-os ao aumento da permeabilidade(18). Os membros desta família atuam através de interações com uma família de três receptores de VEGF e dois correceptores conhecidos como neurofilinas. As diferentes combinações dos membros da família com seus correceptores determinam efeitos específicos. No presente estudo foi utilizado o marcador VGFR-1 para avaliar o complexo coroido-escleral de coelhos hipercolesterolêmicos. Sabe-se que o VEGFR-1 está primariamente envolvido na angiogênese ${ }^{(19)}$, localizando-se na superfície de células hematopoiéticas, macrófagos, monócitos e no endotélio vascular ${ }^{(17)}$.

Relatos a respeito do mecanismo molecular responsável pela formação de neovasos sub-retinianos demonstram a fundamental importância da participação do VEGF na fisiopatogenia da DMRI úmi$d a^{(5,13,14)}$. Sabe-se que o VEGF é secretado fisiologicamente pela superfície basal das células do EPR de forma polarizada para a membrana de Bruch, sendo fundamental para manutenção da integridade dos vasos da coriocapilar ${ }^{(20)}$. Vale lembrar que os vasos da coriocapilar são fenestrados e permeáveis o que possibilita a difusão de nutrientes da corrente sanguinea para as camadas externas da retina ${ }^{(20)}$. Em condições patológicas, supõe-se que o VEGF seja secretado pelo ápice das células do EPR chegando mais facilmente ao espaço sub-retiniano. Este fato associado a alterações da MB conduzem a formação da membrana neovascular sub-retiniana (MNSR)(21).

Já foi demonstrado de forma experimental que o aumento do colesterol sérico provoca espessamento da $M B$, redução da fenestração, estreitamento do lúmen e atrofia dos vasos da coriocapilar. O acúmulo de lipídeos na MB foi considerado responsável pelas modificações anatômicas destas estruturas, hipóxia e pelo consequente aumento da expressão do VEGF na coriocapilar ${ }^{(22)}$. Outro estudo experimental também atribuiu à deposição de partículas lipídicas na MB o aumento da imunorreatividade do VEGF na membrana basal das células do EPR, camada plexiforme externa e segmentos internos dos fotorrecepetores ${ }^{(23)}$. No presente estudo, observou-se significativa imunorreatividade ao VEGFR-1 do complexo coroido-escleral

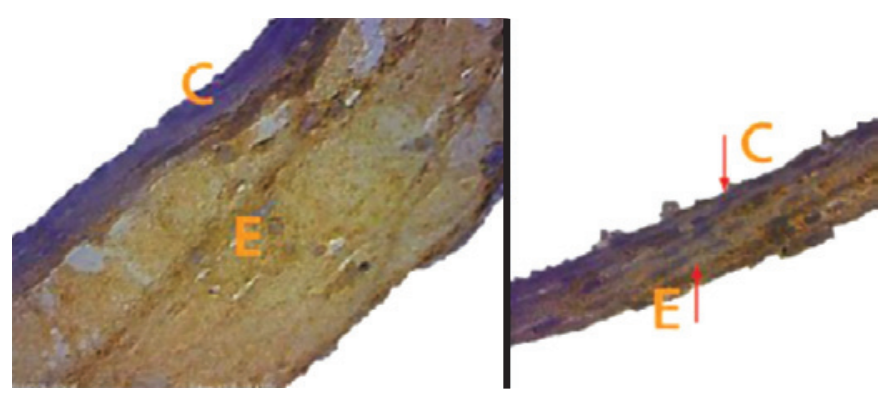

$\mathrm{C}=$ coroide; $\mathrm{E}=$ esclera

Figura 1. Imunorreatividade do complexo coroido-escleral ao RAM-11. A) Complexo coroido-escleral de coelho do GH. Predomínio da coloração acastanhada que indica a alta imunorreatividade ao RAM-11.Grande quantidade de macrófagos na esclera (E). Coroide e esclera espessadas. B) Complexo coroido-escleral de coelho do GN. Predomínio da coloração azul que indica a baixa imunorreatividade ao RAM-11. Coroide e esclera delgadas

Tabela 1. Área total imunorreativa ao anticorpo RAM-11, em micrômetros ${ }^{2}$, obtida com a técnica de morfometria de cores

\begin{tabular}{|c|c|c|c|c|c|c|c|c|}
\hline Variável & Grupo & $\mathbf{n}$ & Média & Mediana & Mínimo & Máximo & Desvio padrão & Valor de $\mathbf{p}^{*}$ \\
\hline \multirow[t]{2}{*}{ RAM-11 } & $\mathrm{GH}$ & 13 & 15835,6 & 15565,2 & 3618,3 & 30073,7 & 6924,9 & \\
\hline & GN & 8 & 2407,6 & 2514,9 & 385,9 & 3761,9 & 1094,8 & $<0,001$ \\
\hline
\end{tabular}

$\mathrm{GH}=$ grupo dieta rica em colesterol; $\mathrm{GN}=$ grupo dieta normal.

${ }^{*}=$ teste $t$ de Student para amostras independentes, $\mathrm{p}<0,05$ 
Tabela 2. Área total imunorreativa ao anticorpo VEFGR-1, em micrômetros ${ }^{2}$, obtida com a técnica de morfometria de cores

\begin{tabular}{lcccccccc}
\hline Variável & Grupo & $\mathbf{n}$ & Média & Mediana & Mínimo & Máximo & Desvio padrão & Valor de $\mathbf{p}$ \\
\hline VEGFR-1 & GH & 13 & 61559406 & 58728731 & 35881479 & 94418194 & 17402678 \\
& GN & 8 & 28770288 & 24866275 & 18942711 & 52270525 & 13368328 & 0,002 \\
\hline
\end{tabular}

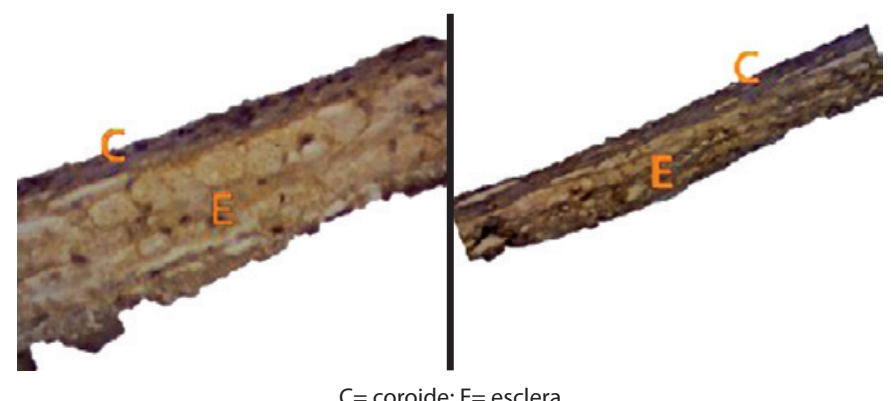

$\mathrm{C}=$ coroide $\mathrm{E}=$ esclera

Figura 2.Imunorreatividade do complexo coroido-escleral aoVGFR-1. A) Fotomicrografia de segmento coroido-escleral de animal do grupo hipercolesterolêmico (GH). As áreas de imunopositividade para o anti-VEGFR-1 coram-se em castanho e estão localizadas no citoplasma de histiócitos espumosos agrupados na esclera. B) Fotomicrografia de segmento coroido-escleral de animal do grupo dieta normal (GN). Observam-se mínimas áreas de imunopositividade para o anti-VEGFR-1. Predomínio da coloração azulada.

do grupo que recebeu a dieta hipercolesterolêmica em relação ao GN ( $p<0,001)$. Sabe-se que além das células do EPR, os macrófagos infiltrados na MNSR secretam grande quantidade de VEGF(24). Estudos experimentais demonstraram que o aumento do colesterol sérico provoca aumento da concentração de macrófagos no complexo coroido-escleral(25,26). No presente estudo observou-se significativo aumento da imunorreatividade ao RAM-11 do complexo coroido-escleral dos animais do GH em relação ao GN $(p<0,001)$. RAM-11 é um anticorpo monoclonal, marcador de macrófagos tecidual. Este fato indica que a elevação do colesterol sérico provocou aumento da concentração de macrófagos nestas estruturas oculares, coincidindo com outros relatos ${ }^{(25,26)}$. Sendo assim, a significativa imunorreatividade ao VEGFR-1 observada no complexo coroido-escleral do GH pode estar relacionada também com a grande quantidade de macrófagos presente nestas estruturas.

Já foi relatada na literatura a elevação do VEGF plasmático em pacientes hipercolesterolêmicos, sendo que o tratamento da dislipidemia provocou a sua redução(27). Este estudo fortalece a idéia de que a DMRI e a aterosclerose compartilham mecanismos fisiopatogênicos similares, e que a hipercolesterolemia é uma fator modificável da DMRI ${ }^{(9,12)}$. Adicionalmente, os estudos experimentais, incluindo o presente relato, que visaram simular a DMRI humana, utilizaram o aumento do colesterol sérico para provocar deposição lipídica na MB e aumento da expressão do VEGF(22,23,28).

Ao escolher coelhos para esta pesquisa, foram ponderadas as vantagens desse animal sobre os demais, que incluem mais disponibilidade, baixo custo (em relação a camundongos transgênicos deficientes dos receptores para o colesterol LDL ou apoliproteína E) e a caracterização genética mais definida ${ }^{(29)}$. Do ponto de vista arterial, a dieta hipercolesterolêmica induz rapidamente a disfunção endotelial e a resposta semelhante à aterogênese, servindo como bom modelo para as doenças cardiovasculares ${ }^{(15)}$. Por isto, a utilização de coelho neste estudo ganhou preferência em relação a outros animais. Sabese também que níveis séricos normais de colesterol variam de 25 a 60 mg\% em contraste com o ser humano, que apresenta variação de 100 a 200 mg\%. Sendo assim, o sistema metabólico do coelho pode ser facilmente sobrecarregado com simples dieta diária hipercoles- terolêmica, tornando os experimentos mais viáveis e reprodutíveis ${ }^{(15)}$. Este fato foi constatado no presente relato. O colesterol do $\mathrm{GH}$ passou de $41,3 \mathrm{mg} / \mathrm{dL}$ para aproximadamente $2146,8 \mathrm{mg} / \mathrm{dL} \mathrm{mg} \%$ no momento da eutanásia.

Em relação a pesquisas oftalmológicas, vários estudos experimentais foram realizados em coelhos com o objetivo de demonstrar que a dieta rica em colesterol provoca anormalidades da esclera, coroide e retina. Tais estudos caracterizaram-se pela administração de dieta rica em colesterol a 0,5\% durante o período mínimo de seis meses ${ }^{(25,30)}$. No presente experimento, a dosagem de colesterol administrada foi mais alta que em outros relatos (colesterol 1\%). Essa dosagem de colesterol permitiu obter alterações imuno-histoquímicas do complexo coroido-escleral nesse período (oito semanas). Esse fato ganha importância em nosso meio científico, pois ficou evidenciado que as alterações na parede ocular de coelhos, assim como nas artérias ${ }^{(15)}$, podem ser obtidas em curto espaço de tempo, facilitando os experimentos e reduzindo os custos das pesquisas. É importante ressaltar que o GN foi submetido à eutanásia em quatro semanas. Este curto período foi suficiente para demonstrar que os exames laboratoriais (colesterol total, triglicerídeos, HDL colesterol e glicemia de jejum) mantiveram-se estáveis, não interferindo na condição de normalidade da análise imuno-histoquímica da coroide e esclera destes animais. Esta decisão foi baseada em estudo anterior ${ }^{(26)}$.

\section{CONCLUSÃO}

É importante frisar que, assim como a aterosclerose, a DMRI em humanos difere nos modelos animais. Desta forma, os achados histopatológicos, imuno-histoquímicos assim como as respostas terapêuticas realçadas em experimento animal muitas vezes não são transponíveis para humanos, devido às peculiaridades fisiopatogênicas como, por exemplos, fenômenos trombogênicos, atividade inflamatória e proliferativa do vaso, assim como características genéticas e fenotípicas.

\section{AGRADECIMENTOS}

À Profa. Dra. Márcia Orlandoswki, responsável pelo Departamento de Estatística da PUC-PR.

\section{REFERÊNCIAS}

1. Friedman DS, O'Colmain BJ, Muñoz B, Tomany SC, McCarty C, de Jong PT, Nemesure B, Mitchell P, Kempen J; Eye Diseases Prevalence Research Group. Prevalence of age-related macular degeneration in the United States. Arch Ophthalmol [Internet]. 2004[cited 2010 Jul 21];122(4):564-72. Erratum in: Arch Ophthalmol. 2011;129(9):1188. Comment in: JAMA. 2004;291(15):1900-1. Available from: ttp:// archopht.jamanetwork.com/article.aspx?articleid $=416232$

2. Ambati J, Ambati BK, Yoo SH, lanchulev S, Adamis AP. Age-related macular degeneration: etiology, pathogenesis, and therapeutic strategies. Surv Ophthalmol [Internet] 2003[cited 2010 Nov 21];48(3):257-93. Available from: http://www.sciencedirect. com/science/article/pii/S0039625703000304

3. Pauleikhoff D, Harper CA, Marshall J, Bird AC. Aging changes in Bruch's membrane: a histochemical and morphologic study. Ophthalmology.1990;97(2):171-8.

4. Ramrattan R, van der Schaft TL, Mooy CM, de Bruijn WC, Mulder PG, de Jong PT. Morphometric analysis of Bruch's membrane, the choriocapillaris, and the choroid in aging. Invest Ophthalmol Vis Sci [Internet]. 1994[cited 2010 Apr 21];35(6):2857-64. Available from: http://www.iovs.org/content/35/6/2857.long

5. Nowak JZ. Age-related macular degeneration (AMD): pathogenesis and therapy. 
Pharmacol Rep [Internet]. 2006 [cited 2010 Jul 21];58(3):353-63. Available from: http:// www.if-pan.krakow.pl/pjp/pdf/2006/3_353.pdf]

6. Guidry C, Medeiros NE, Curcio CA. Phenotypic variation of retinal pigment epithelium in age-related macular degeneration. Invest Ophthalmol Vis Sci [Ínternet]. 2002[cited 2011 Oct 15]; 43(1):267-73. Available from: http://www.iovs.org/content/43/1/267.long

7. Lutty G, Grunwald J, Majji AB, Uyama M, Yoneya S. Changes in choriocapillaris and retinal pigment epithelium in age-related macular degeneration. Mol Vis [Internet]. 1999[cited 2011 Mar 21];5:35. Available from: http://www.molvis.org/molvis/v5/a35/

8. Korte GE, Reppucci V, Henkind P. RPE destruction causes choriocapillary atrophy. Invest Ophthalmol Vis Sci [Internet]. 1984 [cited 2012 Apr 21]; 25:1135-45. Available from: http://www.iovs.org/content/25/10/1135.long

9. Friedman E. The role of the atherosclerotic process in the pathogenesis of age-related macular degeneration. Am J Ophthalmol [Internet]. 2000[cited $2010 \mathrm{Jul}$ 21];130(5):658-63. Comment on: Am J Ophthalmol. 1997;124(5):677-82. Available from: http://goo.gl/G44rG

10. Fisher R. The influence of age on some ocular basement membranes. Eye (Lond) [Internet]. 1987[cited 2012 Aug 25];1(Pt 2):184-9. Available from: http://www.nature. com/eye/journal/v1/n2/abs/eye198735a.html

11. Shweiki D, Itin A, Soffer D, Keshet E. Vascular endothelial growth factor induced by hypoxia may mediate hypoxia-initiated angiogenesis. Nature. 1992; 359(6398): 843-5.

12. Torres RJ, Maia M, Muccioli C, Winter G, Souza GK, Pasqualotto LR, et al. [Modifiable risk factors for age-related macular degeneration]. Arq Bras Oftalmol [Internet] 2009[cited 2010 Feb 21];72(3):406-12. Portuguese. Available from: http://www.scielo. $\mathrm{br} / \mathrm{pdf} / \mathrm{abo} / \mathrm{v} 72 \mathrm{n} 3 / \mathrm{v} 72 \mathrm{n} 3 \mathrm{a} 27 . \mathrm{pdf}$

13. Krzystolik MG, Afshari MA, Adamis AP, Gaudreault J, Gragoudas ES, Michaud NA, et al. Prevention of experimental choroidal neovascularization with intravitreal antivascular endothelial growth factor antibody fragment. Arch Ophthalmol [Internet] 2002[cited 2012 Jun 21];120(3):338-46. Available from: http://archopht.jamanetwork. com/article.aspx?articleid $=269871$

14. van Wijngaarden $P$, Coster DJ, Williams KA. Inhibitors of ocular neovascularization: promises and potential problems. JAMA. 2005;293(12):1509-13.

15. Sun YP, Lu NC, Parmley YW, Hollenbeck CB. Effects of cholesterol diets on vascular function and atherogenesis in rabbits. Proc Soc Exp Biol Med. 2000; 224(3):166-171.

16. Carmeliet P. Angiogenesis in health and disease. Nat Med [Internet]. 2003[cited 2010 Jun 21];9(6):653-60. Available from: http://www.nature.com/nm/journal/v9/n6/full/ nm0603-653.html

17. Ferrara N, Gerber HP, LeCouter J. The biology of VEGF and its receptors. Nat Med [Internet]. 2003[cited 2010 jul 12];9(6):669-76. Available from: http://www.nature. $\mathrm{com} / \mathrm{nm} /$ journal/v9/n6/full/nm0603-669.html

18. Dvorak HF, Nagy JA, Feng D, Brown LF, Dvorak AM. Vascular permeability factor/ vascular endothelial growth factor and the significance of microvascular hyperpermeability in angiogenesis. Curr Top Microbiol Immunol. 1999;237:97-132.

19. Yancopoulos GD, Davis S, Gale NW, Rudge JS, Wiegand SJ, Holash J. Vascular-specific growth factors and blood vessel formation. Nature [Internet]. 2000; [cited 2010 Jun 21]407(6801):242-8. Available from: http://www.nature.com/nature/journal/v407/ n6801/full/407242a0.html
20. Marneros AG, Fan J, Yokoyama Y, Gerber HP, Ferrara N, Crouch RK, et al. Vascular endothelial growth factor expression in the retinal pigment epithelium is essential for choriocapillaris development and visual function. Am J Pathol [Internet]. 2005 [cited 2011 Jun 21];167(5):1451-9. Available from: http://www.ncbi.nlm.nih.gov/pmc/ articles/PMC1603772/

21. Yamada H, Yamada E, Kwak N, Ando A, Suzuki A, Esumi N, et al. Cell injury unmasks a latent proangiogenic phenotype in mice with increased expression of FGF2 in the retina. J Cell Physiol. 2000;185(1):135-42.

22. Schmidt-Erfurth U, Rudolf M, Funk M, Hofmann-Rummelt C, Franz-Haas NS, Aherrahrou $Z$, et al. Ultrastructural changes in a murine model of graded Bruch membrane lipoidal degeneration and corresponding VEGF164 detection. Invest Ophthalmol Vis Sci [Internet]. 2008[cited 2012 Jan 12]; 49:390-8. Available from: http://www.iovs.org/ content/49/1/390.long

23. Rudolf M, Winkler B, Aherrahou Z, Doehring LC, Kaczmarek P, Schmidt-Erfurth U. Increased expression of vascular endothelial growth factor associated with accumulation of lipids in Bruch's membrane of LDL receptor knockout mice. Br J Ophthalmol [Internet]. 2005[cited 2009 Oct 21];89(12):1627-30. Comment in: Br J Ophthalmol. 2005;89(12):1549-51. Available from: http://bjo.bmj.com/content/89/12/1627.long

24. Oh H, Takagi H, Takagi C, Suzuma K, Otani A, Ishida K, et al. The potencial angiogenic role of macrophages in the formation of choroidal neovascular membranes. Invest Ophthalmol Vis Sci [Internet]. 1999[cited 2010 Jun 21];40(9):1891-8. Available from: http://www.iovs.org/content/40/9/1891.long

25. Salazar JJ, Ramírez Al, de Hoz R, Rojas B, Ruiz E, Tejerina T, et al. Alterations in the choroid in hypercholesterolemic rabbits: reversibility after normalization of cholesterol levels. Exp Eye Res [Internet]. 2007[cited 2010 Jun 21];84(3):412-22. Available from: http://www.sciencedirect.com/science/article/pii/S0014483506004192

26. Torres RJ, Maia M, Noronha L, Farah ME, Luchini A, Brik D, et al. Evaluation of choroid and sclera early alterations in hypercholesterolemic rabbits: histologic and histomorphometric study. Arq Bras Oftalmol [Internet]. 2009[cited 2010 Nov 21]; 72(1):68-74. Portuguese. Available from: http://www.scielo.br/pdf/abo/v72n1/v72n1a14.pdf

27. Blann AD, Belgore FM, Constans J, Conri C, Lip GY. Plasma vascular endothelial growth factor and its receptor Flt-1 in patients with hyperlipidemia and atherosclerosis and the effects of fluvastatin or fenofibrate. Am J Cardiol [Internet]. 2001 [cited 2010 Apr 21];87(10):1160-3. Available from: http://www.sciencedirect.com/science/article/pii/ S0002914901014862

28. Moreira EF, Larrayoz IM, Lee JW, Rodriguez IR. 7-Ketocholesterol is present in lipid deposits in the primate retina: potential implication in the induction of VEGF and CNV formation. Invest Ophthalmol Vis Sci [Internet]. 2009[cited 2010 Apr 23]; 50(2):523-32. Available from: http://www.ncbi.nlm.nih.gov/pmc/articles/PMC2811433/

29. Kantor B, Ashai K, Holmes DR Jr, Schwartz RS. The experimental animal models for assessing treatment of restenosis. Cardiovasc Radiation Med [Internet]. 1999 [cited 2010 Jan 21];1(1):48-54. Available from: http://www.sciencedirect.com/science/ article/pii/S1522186598000055

30. Triviño A, Ramírez Al, Salazar JJ, de Hoz R, Rojas B, Padilla E, et al. A cholesterol-enriched diet induces ultrastructural changes in retinal and macroglial rabbit cells. Exp Eye Res [Internet]. 2006 [cited 2010 Feb 3];83(2):357-66. Available from: http://www. sciencedirect.com/science/article/pii/S0014483506001436 